\title{
XIX.
}

\section{Ueber Osteogenesis imperfecta ${ }^{1}$ ).}

Beitrag zur Lehre von den foetalen Knochen-Erkrankungen.

Aus dem Pathologisch-Anatomischen Institut des Neuen Allgem.

Krankenhauses Hamburg-Eppendorf.

Prosector: Dr. E. F. Fränkel.)

von

Dr. H. Hildebrandt, Assistenzarzt.

Hierzu Taf. IX und 2 Text-Abbildungen.

Ich war in der Lage, einen Fall einer seltenen foetalen Knochenerkrankung, der sogenannten Osteogenesis imperfecta, genauer untersuchen zu können, und möchte über diese Untersuchungen kurz berichten.

Im Juli d. J. wurde im Eppendorfer Krankenhause auf der Abtheilung des Herrn Dr. Schütz ein Kind geboren, das schon bei der Geburt den Anwesenden dureh die sehr grosse Weichbeit seines Sehädels auffiel. Die Mutter des Kindes war völlig gesund und war auch früher immer gesund gewesen; insbesondere war weder anamnestiseb, noch objectiv irgend ein. Zeichen von Lues nachzuweisen. Kinder batte sie bisher noch nicht gehabt. Das Kind lebte nach der Geburt noch 8 Stunden, starb dann und kam zur Section.

Sectionsbefund: Es handelt sich um ein schön und kräftig entwickeltes Kind mit allen Zeichen der Reife: die Nägel überragen die Fingerkuppen, die Wollhaare sind überall, selbst auf den Schultern geschwunden, Kopfhaare mehrere Centimeter lang, sebr dicht. Nabel-Insertion fast in der Mitte des Bauches. Auffallend ist der Unterschied in der Länge des Rumpfes und der der Extremitäten; während die Maasse des Rumpfes - vom Scheitel bis zum Damm gemessen, $30 \frac{1}{2} \mathrm{~cm}$ - denen eines ausgetragenen Kindes entsprechen, fallen die Extremitäten, besonders die unteren, und hier besonders die Oberschenkel, durch ihre Kürze auf. Der Vergleieb mit einem normalen, ausgetragenen Kind, welches am selben Tage secirt wurde, ergiebt, dass die Rumpflänge des normalen Kindes um $1 \mathrm{~cm}$ kleiner, dagegen die Länge der Extremitäten um 3-4 $\mathrm{cm}$ grösser ist, als bei dem anderen Kind.

1) Nach einem in der biologischen Abtheilung des arztlichen Vereins zu Hamburg gehaltenen Vortrage. 
Virchow's Archiv. Bd. CLVIII.

Taf. IX.

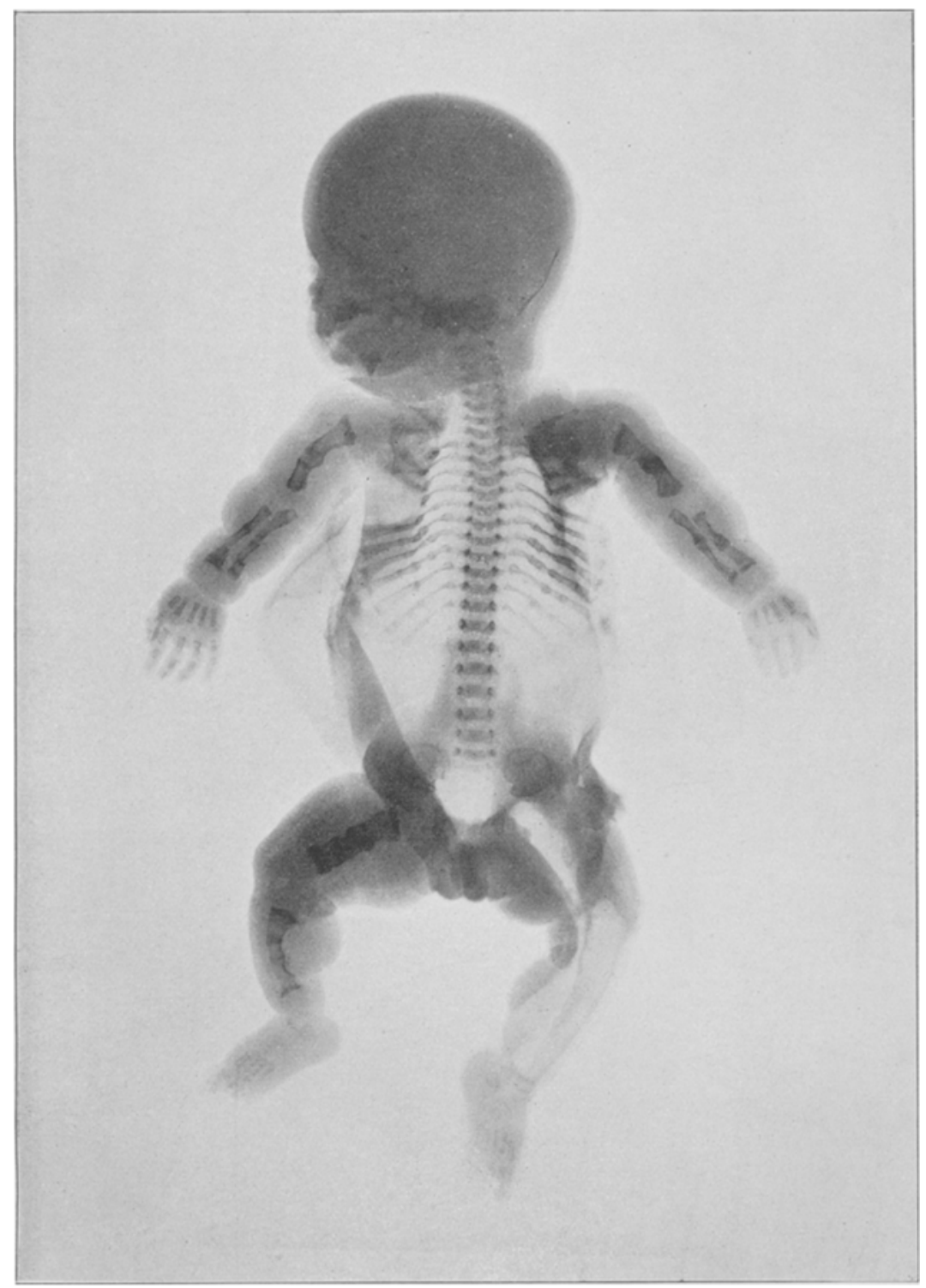

Fig. 1. 
Besonders fallen bei letzterem bei der äusseren Besichtigung einige tiefe Einschnürungen in der Haut der Extremitäten auf, welche sich nicht nur in der Gegend der Gelenke, sondern anch zwischen diesen, besonders deutlich am Unterarm und Unterschenkel, vorfinden; dieselben bilden tiefe, ringförmige Einkerbungen, zu deren beiden Seiten sich das gut entwickelte Fettpolster boch erbebt. Beim Betasten und Bewegen der Extremitäten ist es sebr frappirend, dass man fast gar keinen Widerstand von Seiten der Knochen fühlt. Die Extremitäten sind in ganz abnormer Weise nach allen Richtungen za bewegen und zu biegen, vor Allem in der Gegend der beschriebenen Einkerbungen, so dass man den Eindruck von an diesen Stellen befindlichen Psendarthrosen erbält.

Ein ähnliches Verbalten zeigt der Brustkorb, indem die Rippen eine ausserordentliche Weichbeit und Biegsamkeit darbieten; an den Rippen kann man durch die Haut hindureh eine grosse Anzahl von runden Verdickungen fühlen; es bandelt sich nicht um die bekannten Verdickungen an der KnochenKnorpel-Grenze, den Rosenkranz, vielmehr liegen die Verdickungen weiter nach binten in der eigentlichen Knochensubstanz, und $z$ war meist mehrere bintereinander an derselben Rippe.

Die am meisten auffallenden Verbältnisse zeigt der Schädel; derselbe bietet dem betastenden Finger absolut keinen Widerstand, fühlt sich vielmehr ganz weich und elastisch an, wie ein schlaffer Gummiball. Nur an einigen kleinen Stellen fühlt man ein pergamentäbnliches Knittern bei der Betastung.

Die Besichtigung der inneren Organe bot nichts irgendwie Bemerkenswerthes. Kein Organ zeigte makroskopiseh erkennbare Veränderungen, und, wie ich gleich hier bemerken will, auch die mikroskopische Untersuchung der inneren Organe ergab ein negatives Resultat. In der Leber fanden sich ziemlich zahlreiche, über das ganze Parenchym verbreitete Rundzellen, welche theils einzeln, theils in Häufchen zusammenlagen, weiche aber, wie von Hecker ${ }^{1}$ ) neuerdings betont wurde, nichts Pathologisches und vor Allem Nichts für Lues Charakteristisches sind, sondern sich bei den meisten gesunden Neugeborenen finden. Im Uebrigen zeigten weder die Leber, noch die anderen Organe etwas Auffallendes.

Interessant gestaltete sich die weitere Besichtigung, die genauere Untersuchung des Skelets. Was zunächst die Rippen anlangt, so, sind dieselben an der Knorpel-Knochen-Grenze vielleicht etwas verbreitert, jedoch keineswegs aufgetrieben; wie oben bemerkt, war von aussen bier keine Verdickung fühlbar gewesen. Die im Verlauf der einzelnen Rippen vorbandenen; perlschnurartig angeordneten Verdickungen stellen sich als ächte Knochen-Auftreibungen heraus. Die Rippen sind im Ganzen leicht zu biegen, besonders leicbt an einzelnen dieser Verdickungen; es ist klar, dass es sich hier um Fracturen handelt, welche zum Theil noch sehr wenig, zum Theil besser, unter Bildung eines knöchernen Callus, geheilt sind. Was die Knọchen-

1) Hecker, Beiträge zur Histologie und Pathologie der congen. Syphilis u. s. w. Deutsches Archiv für klinische Medicin 1898, 1. Heft. 
substanz selbst anlangt, so ist dieselbe so mässig entwickelt, dass man die Rippen leicht mit dem Messer schneiden kann. Auf Durchschnitten durch einzelne derselben siebt man, dass die Epiphysen-Linie nicht verbreitert ist, sondern in Form einer scharfen, wenn auch gekrïmmten, meist nach dem Knorpel hin concaven Linie verläuft. Das Knochenmark ist sehr blutreich; an den Fracturstellen sieht man hie und da im Mark sowobl, wie an der Peripherie bläulich-weisse, gleichmässige Fleckchen, welche aus hyalinem Knorpel bestehen. Sämmtliche Rippen zeigen dasselbe Verbalten. Die Anzahl der einzelnen Fracturen ist verschieden; einzelne zeigen 5 Fracturen, die meisten weniger, aber mit alleiniger Ausnahme der letzten, sehr kleinen Rippe ist keine verschont geblieben.

Die Herausnahme der Extremitäten-Knochen ist recht scbwierig; die Knochen sind so weich und so brüchig, bezw. sie sind schon mebrfach gebrochen, so dass es nur mit Mübe gelingt, sie obne weitere Beschädigungen herauszubekommen. Ithr Verbalten ist im Allgemeinen ein ganz gleiches. Vor Allem fällt an ihnen beim ersten Anblick die eigenthümliche Form auf: sie sind sehr kurz und unverhältnissmässig dick; die Dicke ist in der Mitte genau dieselbe, wie an den Enden, so dass die Knochen ein plumpes, walzenförmiges Aussehen baben. Sodann zeigen auch sie zum. Theil ganz frische Brüche ohne reactive Veränderungen, zum Theil dicke Auftreibungen, welche als gebeilte oder in Heilung begriffene Fracturen aufzufassen sind. Die Knochen sind an den Bruchstellen verschiedentlich geknickt, zeigen aber auch im Ganzen starke Verkrümmungen. Die Biegsamkeit ist sehr gross, die Knochensubstanz so weich, dass man auch diese dicken Knochen unter Anwendung eines gewissen Druckes mit dem Messer schneiden kann. Auf Durchschnitten sieht man, dass die EpiphysenLinien ziemlich gerade verlaufen, jedenfalls ist der Knorpel an der Ossifications-Grenze nicht gewuchert, und setzt sich scharf gegen den Knochen ab. Am Knocben ist von einer typisch gebauten Compacta auch in der Mitte der Diaphyse nichts zu sehen; die Compacta ist angedeutet durch eine dünne Knochenschale, welche in gleicher Dicke überall den Knochen umgiebt. Die Spongiosa ist im Allgemeinen blutreich und in Folge dessen tiefroth gefärbt, jedoch finden sich abwechselnd mit dunkelrothen mehr gelblich gefärbte, grössere Flecken. Ueber die ganze Spongiosa verstreut siud kleine, bläulich-weisse Inseln eingesprengt, welche, wie man schon makroskopisch erkennen kann, aus Knorpel besteben.

Das Schädeldach besteht fast völlig aus einem bindegewebigen Sack, nur an einzelnen Stellen sind ungefähr 1 Pfennig-Stück grosse, papierdünne Knochenscheibchen gebildet, welche wenig fest und mehrfach gebrochen sind.

Sehr schön werden die geschilderten Verbältnisse illustrirt durch die Röntgenphotographien, welche ich beifüge. Fig. 1 auf Taf. IX giebt eine Totalansicht des Kindes auf ein Drittel verkleinert. Man sieht deutlich die zahlreichen Verdickungen 
der Rippen; man erkennt, dass sie durch eine Verdickung des kalkhaltigen Knochengewebes zu Stande gekommen sind. Dieselben Verdickungen finden sich an den Extremitäten; man sieht hier mit Sicherheit, dass es sich um Fracturen handelt, indem man 2. B. an den Armen die verschiedensten Stadien vom ganz frischen Bruch bis zu dem mit knöchernem Callus geheilten beobachten kann. Man erkennt ferner ausser den mannigfachen Knickungen und Verbiegungen die oben beschriebene plumpe Form der Knochen, welche besonders am Oberschenkel stark ausgesprochen ist. (Der Knochen des einen Oberschenkels war bereits herausgenommen, als die Photographie hergestellt wurde). Ueber das Knochengerüst selbst, über das Verhältniss zwischen Compacta und Spongiosa giebt Aufschluss Fig. 2. Dieselbe zeigt

Figur 2.

Figur 3.

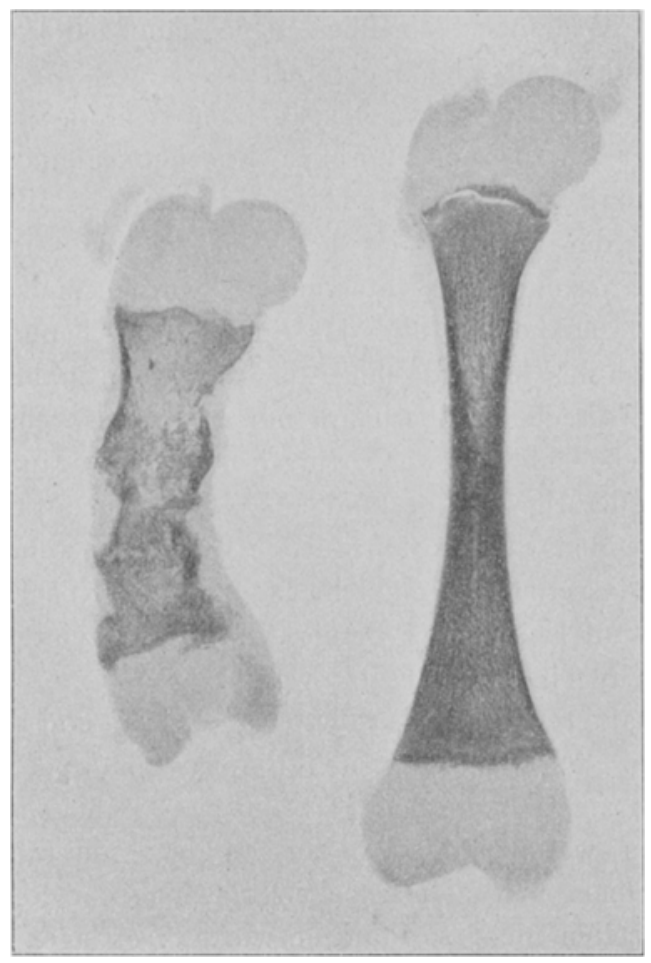


das Schattenbild einer ungefähr $2 \mathrm{~mm}$ dicken Scheibe aus einem Oberschenkel, Zum Vergleich ist in Fig. 3 das Bild einer gleich dicken Seheibe aus dem Schenkel eines normalen Neugeborenen wiedergegeben. Man erkennt die.Verkürzung und Verdickung am Schenkel des kranken Kindes; man sieht, dass die Knochensubstanz im Ganzen sehr mässig entwickelt ist, und dass von einer nur einigermaassen gesetzmässigen Anordnung von Knochenbälkchen keine Rede ist. Von einer Compacta ist kaum eine Andeutung vorhanden; die Spongiosa, welche bei dem normalen Knochen ein sehr regelmässiges, zierliches Gefüge hat, ist bei dem kranken Knochen auch am oberen, nicht durch Fracturen beeinflussten Ende unregelmässig und vor Allem sehr spärlich entwickelt. Die Epiphysenlinie ist im Allgemeinen scharf, wenn auch nicht so regelmässig, wie an dem normalen Knochen. (Der letztere ist in der oberen Epiphysenlinie beim Durchsägen gebrochen). Wie man sich überzeugen kann, sind die Knorpel in beiden Fällen ganz gleich gebildet; sie sind von gleicher Form und Grösse. Deutlich ist dies am oberen Ende; am unteren erscheint der Knorpel des kranken Knochens klejner. Es liegt dies aber daran, dass der Schnitt nicht genau die Mitte getroffen hat, was bei der Krümmuung des Knochens nicht möglich war. Zur mikroskopischen Untersuchung wurden mehrere Rippen, die Extremitätenknochen und das Schädeldach benutzt.

Bezüglich des letzteren fand sich, dass, dem makroskopischen Verhalten entsprechend, äberhaupt nur an einigen wenigen Stellen Knochengewebe gebildet war. Im Grossen und Ganzen war das Schädeldach häutig und bestand aus Zügen von straffem Bindegewebe. An den Stellen, an welchen Knochengewebe vorhanden war, stellte dasselbe eine einfache Lage dünner Knochenblättchen dar, welche an sich nichts Bemerkenswerthes zeigten and mit Osteoblasten regelmässig besetzt waren.

Interessantere Verbältnisse boten die Rippen und die Extremitätenknochen. Was zunächst den Epiphysenknorpel anlangt, so war derselbe ziemlich normal; die einzelnen Zonen des wuchernden Knorpels waren sämmtlich ausgebildet; nur war im Vergleich mit einem normalen Knochen die Zone der Reihenbildung der Knorpelzellen nicht so stark entwickelt: es lagen auch etwas weniger Zellen, 20-30 in einer Reihe, gegen 30-40 in der 
Norm; die Zone der hypertrophischen Knorpelzellen war dagegen etwas breiter, als gewöhnlich; ferner zeigte sich in beiden Zonen eine geringe Unordnung; indessen waren diese Abweichungen von der Norm nicht sehr auffallend, und alle Zonen waren deutlich zu erkennen. Die Epiphysenlinie war nicht gerade, aber sie war scharf abgegrenzt; an einigen Rippen verlief dieselbe bogenförmig, an anderen hatte sie in der Mitte eine scharfe Einknickung, so dass sie einen nach dem Knochen hin gerichteten stumpfen Winkel bildete; die Schenkel des Winkels waren aber verhältnismässig regelmässig. Ein ähnliches Verhalten der Epiphysenlinie habe ich auch bei einigen normalen Rippen gefunden. In der folgenden Schicht, der Schicht der primären Markräume, zeigte sich gegen die Norm eine erhebliche Abweichung. Normalerweise legen sich nach Bildung der primären Markräume sofort epithelähnliche Osteoblasten an die Wände der Markräume an und bilden feine Knochenplättchen, welche sich den stehenbleibenden Resten der Knorpel-Grundsubstanz anfügen. In Gegensatz hierzu findet sich in unserem Falle in der Gegend der primären Markräume keine Spur von Knochensubstanz. Die übrigbleibenden Reste der Knorpel-Grundsubstanz sind ohne Anlagerung von Osteoblasten allseitig direct von Markgewebe umgeben. Diese Reste, anfangs noch ziemlich regelmässig, werden mehr und mehr eingeschmolzen, und liegen dann ganz unregelmässig zerstreut umber. Erst in einer ziemlich grossen Entfernung vom Knorpel beginnt eine spärliche Anlagerung von Knochensubstanz an diese unregelmässigen Inseln. Die Knochenbildung bleibt auch jetzt noch gering, die Knorpel-Grundsubstanzreste überwiegen erheblich und setzen sich weit in den älteren Kuochen hinein fort. So entsteht ein Gewebe, welches in der Hauptsache aus Markräumen mit zahlreichen Gefässen besteht, $z$ wischen welchem eingestreut sich sehr spärliche, dünne Knochenbälkchen, meist mit einem eingelagerten Kern von Knorpel-Grundsubstanz, befinden. Die Knochenbälkchen liegen sehr uaregelmässig, ihre Substanz zejgt keinen lamellösen Bau, wie er nach Kölliker ${ }^{1}$ ) dem normalen endochondralen, auf Knorpelreste abgelagerten Knochen zukommt, sondern es handelt sich um eine sehr grobfaserige Substanz; dieselbe enthält auch viel zahlreichere

1) Kölliker, Handbuch der Gewebebelehre des Menschen, Leipzig. 
Kerne, welch' letztere grösser sind, nicht die ganz typische Sternform haben und dichter bei einander liegen, als gewöhnlich, an Stellen, an welchen Brüche stattgefunden haben, befinden sich sowohl in der Peripherie, als im Centrum grosse Inseln von byalinem Knorpel, welche makroskopisch schon zu sehen waren. Am Rande dieser Knorpelinseln erkennt man den directen Uebergang der Knorpelzellen in Knochenzellen. Da normalerweise beim Callus diese Metaplasie von Knorpel- und Knochengewebe sich findet, so ist dieser Befund auch hier leicht begreiflich, denn die Knorpelinseln sind als knorpeliger Callus, in Folge der Fracturen neu entstanden, aufzufassen.

Einen sehr auffallenden Befund bilden noch zahlreiche, in sämmtlichen Knochen sich vorfindende, nekrotische Stellen. Dieselben sind unregelmässig zerstreut in der Nähe der Epiphyse sowohl, wie in der Mitte des Knochens, unterhalb des Periosts, wie mitten im Markgewebe. Sie bilden grössere oder kleinere homogen aussehende Plaques, welche ohne jeden Uebergang sich direct in gesundes Gewebe fortsetzen. An der Nekrose sind alle Gewebsarten, Markgewebe, Knorpel und Knochen betheiligt. Einzelne Knochen- und Knorpelstïckchen sind theilweise in die nekrotischen Stellen eingelagert; dieser Theil ist dann selbst nekrotisch, während der andere Theil desselben Bälkchens, welcher im Gesunden liegt, normales Verhalten zeigt. Am Rande eines solchen nekrotischen Heerdes finden sich, den abgestorbenen Knochenbälkchen oder Knorpelstückchen anlagernd, zahlreiche Riesenzellen, welche als Fremdkörper-Riesenzellen aufzufassen und damit beschäftigt sind, die Fortschaffung der abgestorbenen festen Gewebe zu besorgen.

Nachzutragen ist noch, dass den Knochenbälkchen meist eine Lage zierlicher Osteoblasten anliegt, dass sich aber auch sehr zahlreiche Osteoklasten in Lacunen liegend finden.

Was den Kalkgehalt der Knochen anlangt, so lässt sich schon aus der grossen Weichheit derselben schliessen, dass der Kalkgehalt erheblich vermindert ist. Wie weich die Knochen sind, lässt sich auch daraus entnehmen, dass Knochenstückchen, welche nur einige Tage in Müller'scher Lösung gelegen hatten, sich ganz gut, ohne Schaden für das Messer, mit dem Mikrotom schneiden liessen. Aber auch durch die mikroskopische Be- 
trachtung derartiger Schnitte lässt sich sicher eine Verminderung des Kalkgehaltes nachweisen. Besonders deutlich ist dies an der primären Verkalkungszone, indem sich Kalkkörnchen nur ganz am untersten schmalen Rand der hypertrophischen Knorpelzellen vorfinden, so dass die primäre Verkalkungszone auf ein Minimum reducirt erscheint.

Was die Classifieirung des geschilderten Krankheitsbildes anlangt, so handelt es sich um einen derjenigen Fälle, welche mit dem Namen Osteogenesis imperfecta belegt sind und früher allgemein der Rachitis foetalis zugerechnet wurden. Wegen der ringförmigen Auftreibungen an den Fracturstellen wurde die Krankheit als Rachitis foetalis annularis bezeichnet.

Die foetale Rachitis spielte in früheren Zeiten überhaupt eine grosse Rolle; die meisten Knochen-Erkrankungen der Neugeborenen wurden als Rachitis gedeutet. Erst in neuerer Zeit hat man gelerut, einzelne wohl charakterisirte Krankheitsbilder von der Rachitis zu trennen and die letztere auf einen kleineren Rahmen zu beschränken. Doch hat die Lehre von der foetalen Rachitis manchen Wechsel erfahren, ehe man auf dem heutigen Standpunkt ankam. Eine genauere Schilderung der Lehre von der foetalen Rachitis mit Literatur-Angaben findet sich bei $\mathrm{J}_{0}$ hannessen ${ }^{1}$ ).

Es erschienen schon in den 70er Jahren Arbeiten, welwhe sich dagegen aussprachen, dass die bisher als Rachitis gedeuteten foetalen Knochen-Erkrankungen, welche sich besonders durch kurze, plumpe Extremitäten auszeichneten, wirklich rachitischen $\mathrm{Ur}_{\mathrm{r}}$ sprungs seien. In der folgenden Zeit wurden immer mehr Stimmen laut, welche dieselbe Anschauung vertraten; es wurde eine Reihe vou Fällen veröffentlicht, welche bei genauerer Untersuchung sich als grundverschieden von Rachitis herausgestellt hatten. Mit Hülfe des Mikroskops wurde der Knorpel als Sitz dieser Erkrankung erkannt, und es wurden nun von den einzelnen Autoren die verschiedensten Namen für diese bestimmte Erkrankung vorgeschlagen. Einen vorläufigen Abschluss fauden die Untersuchungen durch die bekannte Monographie Kaufmann's ${ }^{2}$ ).

1) Johannessen. Chondro-Dystrophia foetalis hyperplastica. Ziegler's Beiträge Band 23. 1898.

2) Kaufmann, Untersuchungen über die sogenannte foetale Rachitis (Chondro-Dystrophia foetalis) Berlin $\mathbf{1 8 9 2 .}$ 
Derselbe beschreibt in seiner Arbeit ausführlich die stattliche Anzahl von 13 Fällen, welche er selbst zu untersuchen Gelegenheit hatte, und bespricht die bis dahin erschienene Literatur. Auf Grund seiner Untersuchungen kommt er zu dem Resultat, dass es eine ziemlich häufige Skelet-Erkrankung der Neugeborenen giebt, welche mit Rachitis gar nichts zu thun hat; dieselbe tritt in verschiedenen Formen auf, aber alle haben das Gemeinsame, dass es sich um eine primäre Erkrankung des Knorpels handelt, dessen Wachsthum in verschiedener Weise gestört ist. Kaufmann bezeichnet die Krankheit mit dem Namen Chondro-Dystrophia foetalis und stellt 3 verschiedene Formen auf: die malacische, die hypertrophische und die hypotrophische.

Durch die erwähnten Arbeiten wurde eine grosse Reihe von Fällen, welche früher der foetalen Rachitis zugezählt waren, von der letzteren abgeschieden und die foetale Rachitis erbeblich eingeschränkt.

Die im Jahre 1882 und 1885 erschienenen Arbeiten von Kassowitz ${ }^{1}$ ) waren geeignet, in anderem Sinne eine Wendung in der Lehre von der foetalen Rachitis hervorzubringen. Auf Grund von umfassenden Untersuchungen stellte derselbe nehmlich die Theorie auf, die Rachitis sei nicht, wie bisher angenommen, lediglich auf die äusseren Einflüsse zurückzuführen, welchen das Kind von seinem ersten Lebenstage ausgesetzt sei, sondern schon während des foetalen Lebens sei der Keim der Krankheit bei den Kindern vorhanden und er entwickle sich im Laufe der Zeit mehr und mehr. Er stützt sich bei Aufstellang dieser Lehre auf Untersuchungen einer grossen Zahl von Neugeborenen, von denen er 89,5 pCt. als mit Rachitis behaftet gefunden hatte. Die Diagnose wurde von ihm nicht immer mikroskopisch gestellt, sondern stützte sich in vielen Fällen auf bestimmte äussere, der Rachitis eigenthümliche Merkmale. Die Befunde wurden in den nächsten Jahren mehrfach nachgeprüft, einzelne Autoren bestätigten die Kassowitz'schen Angaben, andere kamen zu etwas abweichenden Resultaten, indem sie nur bei 60 bis 50 pCt. angeborene Rachitis constatirten, wieder andere dagegen,

1) Kassowitz, die norrnale Ossification und die Erkrankungen des Knochensystems bei Rachitis und heriditärer Syphilis. Wien 1882 u. 1885 . 
wie $Q u i s l i n g^{1}$ ) und Lentz ${ }^{2}$ ) fanden viel geringere Zahlen, nehmlich nur $11 \mathrm{pCt}$. Die Verschiedenheit dieser Resultate veranlasste nun Tschistowitsch ${ }^{3}$ ) zu einer sehr eingehenden Arbeit über diesen Gegenstand; er veröffentlichte im vorigen Jahre das Resultat seiner zahlreichen Untersuchungen. Er kritisirt vor Allem die von Kassowitz angewendete Methode, Rachitis nur nach äusseren Merkmalen zn diagnosticiren, da die angeführten äusseren Zeichen nicht für Rachitis charakteristisch seien. Für einzig maassgebend hält er die mikroskopische Untersuchung, wie sie auch von Lentz ausschliesslich angewendet wurde. Tschistowitsch fand bei 100 Neugeborenen in 72 Fällen völlige normale Osteogenese, 15 Fälle stellten sich als typische syphilitische Knochen-Erkrankungen heraus, 13 Fälle waren theils syphilis- theils rachitis-verdächtig.

Es folgt aus diesen Untersuchungen, welche sich auf eine feste Grundlage, den mikroskopischen Befund, stïtzen, dass die Kassowitz'schen Angaben von der Häufigkeit der intranterinen Rachitis nicht aufrecht zu erhalten sind; es folgt ferner daraus, dass ein grosser Theil der Fälle, welche äusserlich den Eindruck von Rachitis machen, nicht rachitischen Ursprungs sind, sondern sich mikroskopisch als syphilitische Erkrankungen feststellen lassen.

Es ist somit durch die Arbeit von Tschistowitsch der foetalen Rachitis nicht nur wieder der Platz angewiesen, den sie vor den Kassowitz'schen Veröfentlichungen inne hatte, sondern durch Abscheidung der syphilitischen Erkrankungen ist sie sogar noch erheblich weiter eingeschränkt worden.

Im Jahre 1889 endlich berichtete Stilling ${ }^{4}$ ) über einen Fall einer eigenthümlichen Knochen-Erkrankung, welche sich durch die Menge von intrauterin entstandenen Knochenbrüchen auszeichnete. Da auch der mikroskopische Befund von dem bei den übrigen foetalen Knochen-Erkrankungen üblichen abwich, so

1) Quisling. Studien über Rachitis. Archiv für Kinderheilkunde. 1888.

2) Lentz. Osteochondritis syphilitica und Rachitis congenita. J. D. Göttingen 1895.

3) Tschistowitsch. Zur Frage von der angeborenen Rachitis. Dieses Archiv. Bd. 148. 1897.

4) Stilling, Osteogenesis imperfecta. Dies. Archiv, Bd. 115, 1889. 
stellte er diese Erkrankungsform als eine selbständige auf, sonderte sie von der foetalen Rachitis, zu der man sie bisher rechnete, $a b$ and bezeichnete sie mit dem schon früher von $V_{r o l i k}{ }^{2}$ ) gebrauchten Namen Osteogenesis imperfecta.

Dass auch ächte Rachitis intrauterin vorkommt, ist zunächst theoretisch nicht von der Hand zu weisen, es sind aber auch Fälle davon beobachtet worden. Tschistowitsch, welcher in seiner 3. Gruppe rachitis-ähnliche Fälle beschreibt, erkennt das an. Auch Paltauf ${ }^{2}$ ) berichtet über ächte Rachitis bei Neugeborenen.

So seben wir, dass die Lehre von der foetalen Rachitis im Laufe der Zeit manche Wandlung durchgemacht hat, dass die foétale Rachitis zu Zeiten eine grosse Rolle in der Pathologie spielte, dass sie aber jetzt auf einen kleinen Raum beschränkt worden ist und eine offenbar recht wenig häufige Erkrankung darstellt.

Wir köunen nach dem Gesagten die foetalen KnochenErkrankungen eintheilen in 1. ächte Rachitis, 2. Chondro-Dystrophia foetalis mit ihren drei Unterabtheilungen, 3. syphilitische KnochenErkrankungen, 4. Osteogenesis imperfecta. ${ }^{\top}$.

Was die bisherigen Veröffentlichungen über die letztere, uns besonders interessirende Krankheitsform anlangt, so sind dieselben recht spärlich. Stilling, welcher, wie erwähnt, den letzten Fall im Jahre 1889 beschrieb, bringt eine Zusammenstellung der bis dahin beobachteten Fälle, zusammen 8; ausserdem erwähnt Paltauf in seiner Monographie über den Zwergwuchs kurz 3 fremde und 2 eigene Beobachtungen. Allen Fällen gemeinsam sind die grosse Weichheit der Knochen, die zahlreichen Fracturen und die dadurch hervorgerufenen Verunstaltungen der Extremitäten. Die makroskopische Beschreibung ist in einzelnen Fällen sehr sorgfältig; sie zeigen die grösste Uebereinstimmung mit dem Stilling'schen Fall, wie mit dem unsrigen. Mikroskopisch untersucht sind die früheren Fälle nicht. Die ersten und einzigen diesbezüglichen Untersuchungen stammen von Stilling.

Derselbe benutzte zur mikroskopischen Untersuchung das

2) Cit. nach Stilling (a. a. 0.)

3) Faltauf. Ueber den Zwergwuchs in anatomischer u. s. w. Beziehung. Wien 1891. 
Schädeldach, sowie eine Tibia und einige Rippen. Im Schädeldach fand er nur an einigen kleinen Stellen spärliche Knochenbälkchen entwickelt. Dieselben wichen in ihrer Structur nicht von der normaler kindlicher Knochen ab. Bei Beschreibung der Röhrenknochen betont Stilling vor allem das normale Verhalten des Knorpels, an welchem sämmtliche Zonen gut ausgebildet waren, sowie das der Knochen-Knorpelgrenze, welche in gerader Richtung ohne Unregelmässigkeit verlief. Bezüglich der Diaphysen schreibt er: „Der weitaus grösste Theil derselben besteht gar nicht aus Knochen, sondern aus einem Markgewebe, in dem man förmlich nur mit Mühe einige der den kindlichen Knochen charakterisirenden geflechtartigen Knochenbälkchen zu Gesicht bekommt. Die spärlichen Knochentheilchen sind verkalkt, einzelne zeigen eive unverkalkte Randzone. Die Mehrzahl der das Gerüst der Tibia zusammensetzenden Pfeiler besteht aus den ausserordentlich breiten Knorpelresten, den Richtungsbalken, welchen sich nur ganz feine Säume jungen Knochengewebes angelegt haben. Dieses auffallende Missverhältniss zwischen Knorpelresten und neugebildetem Knochen findet sich am ausgesprochensten in den an die Ossificationslinie angrenzenden Abschnitten, doch trifft man auch in den mehr central gelegenen Partien der Diaphyse sehr breite Knorpelstücke ohne jede bemerkenswerthe Anlagerung von Knochensubstanz. Etwas besser ist die periostale Knochenbildung, so dass der Knochen, was er überhaupt an Festigkeit besitzt, der allerdings dünnen Schale von Periostknochen verdankt. An den Stellen der Fracturen und Infractionen findet sich Knorpelgewebe, welches zum Theil directen Uebergang in Knochen aufweist."

Dieser Befund stimmt fast genau mit dem von mir oben geschilderten überein.

Die mangelhafte Anbildung von Knochengewebe und das Erhaltensein grosser Inseln von Knorpel-Grundsubstanz bei normalem Verhalten der Knorpel sind die beiden Fällen gemeinsamen und das Krankheitsbild als eigenartiges kennzeichnenden Merkmale. Einige Unterschiede zwischen dem Stilling'schen und unserem Falle bestehen allerdings. Zunächst ist in dem letzteren auch die Knochenbildung von Seiten des Periosts eine so spärliche, dass man kaum von einer Knochenschale reden 
kann. Ein zweiter wichtiger Unterschied ist sodann gegeben in dem Vorhandensein zahlreicher nekrotischer Stellen, welche einen sehr auffallenden Befund bilden. Stilling erwähnt in seiner Beschreibung nichts von derartigen abgestorbenen Partien.

Um den Beweis zu bringen, dass es sich in den geschilderten Fällen wirklich um eine Krankheit eigener Art handelt, ist es nöthig, noch etwas genauer auf das Verhalten des wachsenden Knochens in der Norm und bei pathologischen Zuständen einzugehen und die etwa sich findenden Unterschiede festzustellen.

Bekanntlich geht die enchondrale Knochenbildung folgendermaassen vor sich: Markgewebe mit Gefässen dringt an der Ossifications-Linie in die primären Verkalkungszonen des Knorpels ein und bringt den Knorpel zum grossen Theil zum Schwund; die Knorpel-Kapseln werden eröffnet, die Knorpel-Zellen gehen zu Grunde. So entstehen die primären Markräume, welche getrennt werden von stehenbleibenden Resten der Knorpel-Grundsubstanz.

Schon in diesen primären Markräumen beginnt alsdann die Bildung von Knochen, indem sich an die Wand der Markräume Osteoblasten anlegen, welche feine Lamellen von Knochensubstanz bilden. Die Knorpel-Grundsubstanz, welche in der Gegend der ersten Markräume meist noch ein Gitterwerk bildet, wird weiter aufgelöst, jedoch bleiben längliche, der Richtung des ganzen Knochens parallel verlaufende Streifen bestehen; an diese legen sich immer mehr Knochen-Lamellen an. So entstehen kräftige, im Allgemeinen längs gerichtete Knochenbälkchen, welche in ihrem Innern einen Rest von Knorpel-Grundsubstanz einschliessen. Diese primär gebildeten KnochenBälkchen sind keine bieibenden Bildungen, sondern werden später bei Bildung der Markhöhle wieder resorbirt, und mit ihnen auch die Knorpel-Grundsubstanzreste. Doch hat Kölliker (a.a. 0.) noch bei Sechszehnjährigen einzelne solcher überbleibenden Inseln nachgewiesen.

Die geschilderten Knorpel-Grundsubstanzreste kann man sehon bei den gewöhnlichen Färbe-Methoden ziemlich gut erkennen, sie färben sich z. B. mit Hämatoxylin blau; in einzelnen Fällen versagen die Methoden aber, wie z. B. gerade in dem 
von mir untersuchten Fall. Ich möchte deshalb ein anderes Verfahren zur Darstellung derselben empfehlen. Von der Erwägung ausgehend, dass Knorpel-Gewebe sich sehr intensiv mit polychromem Methylenblau färbt und auch bei starker Entfärbung die Farbe nicht abgiebt, versuchte ich eine Doppel. färbung mit Methylenblau und van Gieson's Farbgemisch. Der Versuch gelang und die Methode giebt gute Bilder. Man verfährt so, dass man zunächst ziemlich intensiv mit Methylenblau vorfärbt und die Entfärbung mit Glycerinäther und Alcohol nicht za stark vornimmt. Die Schnitte werden dann in gewöhnlicher Weise mit Hämatoxylin und Pikrinsäurefuchsin behandelt, abgewaschen und nun in Alcohol differenzirt. Im Alcohol wird jetzt noch sehr viel Methylenblau abgegeben; die Schnitte müssen so lange darin bleiben, bis das Knochen-Gewebe roth geworden ist und makroskopisch kein Methylenblau mehr enthält. In so behandelten Schnitten sind der Knorpel und sämmtliche im Knochen-Gewebe vorhandenen Knorpel-Grundsubstanzreste intensiv blau, das Knochen-Gewebe contrastirt scharf durch seine leuchtend rothe Farbe. Die Kerne der Zellen sind braun, Blutkörperchen u. s. w. gelb. Da das Knorpel-Gewebe sich sehr intensiv blau färbt, so eignet sich die Methode hauptsächlich zu Uebersichts-Bildern.

Von dem geschilderten Modus der normalen Knochenbildung weicht unser Fall in ganz erheblicher Weise ab. Zunächst wird die Knorpel-Grundsubstanz in weniger ausgiebiger Weise resorbirt, so dass grössere Inseln, und diese in grösserer Zahl übrig bleiben, dann aber, und das ist das Bemerkenswertheste, geht die Bildung von Knochen-Lamellen in sehr spärlicher Weise vor sich; nicht schon in den primären Markräumen; sondern ein erhebliches Stück vom Ossifications-Rande entfernt, legen sich die ersten Knochen-Lamellen an die Peripherie der Knorpel-Grundsubstanzreste an. So entsteht zwischen Knorpel und dem ersten Knochen-Bälkchen eine ziemlich breite Zone, in welcher sich nur. Knorpel-Grundsubstanzreste finden. Die weitere Resorption dieser Reste ist eine unregelmässige, so dass nicht parallel zur Längsrichtung gestellte Streifen übrig bleiben, sondero durehaus unregelmässig gestaltete und unregelmässig durcheinanderliegende Inseln. Legen sich nun nachher 
an letztere Knochen-Lamellen an, so bilden auch diese unregelmässig durcheinander liegende Bälkchen. Die Knochenbälkchen selbst sind im Verhältniss zur Norm ausserordentlich dünn und haben ein grobfaseriges Gefüge. Die Ephysen-Linie ist nicht ganz regelmässig, indessen trennt sie scharf Knorpel und Knochen. Der Knorpel selbst ist nicht verdickt, die einzelnen Zonen nur wenig von der Norm abweichend.

Im Vergleich mit der Norm zeigt also unser Fall erhebliche Abweichungen; es fragt sich nun, ob dies auch beim Vergleich mit den übrigen Knochen-Erkrankungen der Fall ist.

Sehen wir zunächst den Befund bei Rachitis genauer an, so werden wir erkennen, dass es sich bei Rachitis um eine ganz andere Erkrankung handelt. Die starke Wucherung des Knorpels, welche sich bei Pachitis findet, die verbreiterte OssificationsGrenze, das ungleichmässige Eindringen des Markgewebes in den Knorpel, endlich die Bildung des charakteristischen, osteoiden Gewebes, alles Erscheinungen, von welchen bei unserem Fall keine Rede ist, scheiden die beiden Krankheiten scharf von einander. Färbt man rachitische Knochen nach der angegebenen Methode, so kann man constatiren, dass auch im Verhalten der Knorpel-Grundsubstanzreste zwischen beiden Krankheiten ein grosser Unterschied besteht. Bei Rachitis findet man die bekannten abgesprengten Inseln von intactem Knorpel mit Knorpe]Zellen in der stark verdickten Ossifications-Zone, nirgends aber findet man isolirte Reste der Knorpel-Grundsubstanz ohne Zellen. Augenscheinlich wird die Knorpel-Grundsubstan\% durch den rachitischen Process langsam völlig aufgelöst, während im Gegentheil die bei normaler Ossification zu Grunde gehenden KnorpelZellen zum grossen Theil erhalten bleiben. Schön kann man bei der Methyleoblau-Fuchsinfärbung den directen Uebergang von Knorpel-Zellen in Kuochen-Zellen durch die einzelnen Stadien verfolgen. Fassen wir eine in der verbreiterten Ossifications-Zone liegende Knorpel-Insel in das Auge, so sehen wir, dass dieselbe, in der Mitte sich intensiv blau färbend, das Verhalten des normalen Knorpels zeigt; am Rande färbt sich dieselbe nicht mehr blau, die grossen, neben einander liegenden Knorpel-Zellen sind durch eine homogene, völlig farblose Zwischensubstanz von einander getrennt; noch etwas weiter entfernt tritt 
streifiges, mit Fuchsin sich stark roth färbendes Gewebe zwischen den Knorpel-Zellen auf: die Knorpel-Zellen betheiligen sich an der Bildung des osteoiden Gewebes; an anderen Stellen umgeben sich die Knorpel-Zellen mit einer runden, intensiv roth sich färbenden, dichten Schale: sie bilden die sogenannten Osteoid-Kugeln; die zwischen mehreren solchen Kugeln gelegene Zwischensubstanz verdichtet sich dann immer mehr, färbt sich ebenfalls intensiv roth, und geht allmählich in wirkliche KnochenBälkchen über.

Ebenso wie der rachitische, zeigt auch der syphilitische Knochen andere Verhältnisse, als der unserige, wenn sich auch nicht lengnen lässt, dass gewisse Aehnlichkeiten bestehen. Ich selbst hatte in der letzten Zeit nur Gelegenheit 2 Fälle von syphilitischer Knochen-Erkrankung zu untersuchen. Die Knochen waren in beiden Fällen sehr stark verändert: es war bereits zu ausgedehnter Zerstörung des Knorpels mit EpiphysenLösung gekommen, so dass an der Ossificationslinie weder Knorpelzellen, noch Knorpel-Gruodsubstanz übrig waren, sich hier vielmehr ein zellreiches Granulationsgewebe befand. In den älteren, nach rückwärts gelegenen Partien zeigten die Knochenbälkchen normales Verhalten, in ihrem Inneren fand sich die bekannte Insel von Knorpel-Grundsubstanz. Zur Zeit ihrer Bildung war offenbar der krankhafte Process noch nicht entwickelt gewesen. Diese sehr weit vorgeschrittenen Fälle sind zum Vergleiche nicht geeignet, und ich halte mich deshalb bei der Beschreibung der syphilitischen Knochenveränderungen an die Darstellung von Tschistowitsch (a. a. 0.); derselbe sagt am Schlusse seiner Beschreibung der luischen Knochen-Affectionen, dass dieselben hauptsächlich durch eine verstärkte, unregelmässige Ablagerung von Kalksalzen in dem hypertrophischen Knorpel, durch eine bedeutende Hemmung in der Entwicklung der Knochenplättchen, durch die Resistenz der verkalkten Knorpelbalken, und schliesslich durch das Auftreten von Granulationsgewebs-Heerden, welche zu regressiven Veränderungen neigen, charakterisirt sind. Die Beschreibung der mangelhaften Knochenbildung und des Stehenbleibens der Knorpel-Grundsubstanzreste hat eine entschiedene Aehnlichkeit mit der unserigen, indessen bietet unser Fall doch so grosse und wichtige Unterschiede, dass man beide Formen 
gut von einander trennen kann. Zunächst, und dass ist von grosser Wichtigkeit, ist von einer stärkeren Kalkablagerung im Knorpel in unserem Falle keine Spur zu erkennen. Dass hiervon keine Rede sein kann, zeigt schon das physikalische Verhalten, die grosse Weichheit des Knochens und Knorpels, die Möglichkeit, ganz leicht die unentkalkten Stückchen su schneiden; dann aber reichte, wie Mikrotomschnitte von nur sehr mangel haft entkalktem Knochen lehrten, die Kalkablagerung im Knorpel nicht nur nicht weiter als gewöhnlich, und war auch nicht unregelmässig, vielmehr entsprach die primäre Verkalkungszone nur dem untersten Saum der letzten hypertrophischen Knorpelzellen. Die Neigung des Knorpels zu verstärkter Kalkablagerung ist nach Lentz und Tschistowitsch eines der am meisten charakteristischen Symptome bei hereditärer Lues, und sie ist so stark ausgeprägt, dass in einzelnen Fällen die primäre Verkalkungszone doppelt, ja dreimal so breit ist, wie unter normalen Verhältnissen. Ausser dem Fehlen dieser starken Verkalkung des Knorpels zeichnet sich unser Fall noch aus durch das Fehlen jener an Gummibildungen erinnernden Granulationsgewebs-Heerde, welche zu regressivem Zerfall, Verfettung u. s. w. neigen. Hiervon ist keine Spur zu sehen. Dahingegen finden sich zahlreiche, ganz anders sich verbaltende nekrotische Partien, welche Knochen, Knorpel und Markgewebe betreffen, und welche gleichmässige homogene Heerde ohne irgendwelche Anhäufung von Zellen in der Umgebung darstellen. Von derartigen Nekrosen erwähnt Tschistowitsch in seinen sämmtlichen Fällen nichts. Ich glaube, dass diese Unterschiede so schwerwiegende sind, dass man trotz einer gewissen Aehnlichkeit doch beide Processe trennen muss, zumal da in unserem Falle weder anamnestisch, noch durch Untersuchung der übrigen Organe irgend etwas für Lues Sprechendes sich hat nachweisen lassen.

Es erübrigt noch, die Chondro-Dystrophia foetalis zu besprechen und auf etwaige Unterschiede hinzuweisen. Sehen wir von der hypertrophischen Form dieser Erkrankung ab, einer Form, von welcher nur 2 Fälle bisher beschrieben wurden, eine von Kauffmann (a.a. 0.) und einer von Johannessen (a. a. 0.), und welche sich wegen der excessiven Wucherung des Knorpels überhaupt nicht mit unserem Fall vergleichen lässt, sehen wir ferner ab von der malacischen Form, bei welcher es sich um 
eine Auflockerung des Knorpels handelt, was bei uns ebenfalls nicht zutrifft, so bleibt nur die hypoplastische Form übrig welche wegen ihrer Häufigkeit auch am meisten Beachtung verdient.

Kaufmann ${ }^{1}$ ) bezeichnet in seinem Lehrbuch als das Wesentliche der Erkrankung eine mangelhafte Knorpelwucherung und Unfähigkeit desselben zur Bildung von Längsreihen. Auch die übrigen Beobachter heben sämmtlich die mangelhafte Ausbildung der typischen Zonen am wachsenden Knorpel, besonders die sehr mangelhafte Reihenbildung hervor. In seiner Monographie über Chondro-Dystr. foet. giebt Kaufmann auf Tafel 5 in Fig. A u. E mikroskopische Bilder dieser Erkrankung; die sämmtlichen Zonen des wachsenden Knorpels sind auf einen ganz schmalen Streifen beschränkt, von einer Reihenbildung der Knorpelzellen ist fast nichts $z u$ erkennen. Das Bild ist ein völlig anderes, als in unserem Falle, in welchem zwar eine gewisse Unregelmässigkeit in den einzelnen Koorpelzonen vorhanden ist, bei welchem aber sämmtliche Zonen in genügender Breite ausgebildet sind, so dass die Knorpel-Veränderung vollständig zurücktritt gegen die mangelhafte Knochen-Entwicklung. Umgekehrt ist in dem Kaufmann'schen Falle, wie ein Blick auf die Abbildungen lehrt, eine normale Bildung von Knochensubstanz vorhanden. Es legen sich in den ersten Markräumen bereits Knochen-Lamellen den Wandungen an. Man kann deshalb wohl mit Recht sagen, dass auch diese beiden Krankheiten nichts mit einander gemein haben.

Es erhellt aus dem Gesagten, dass wir es bei der Osteogenesis imperfecta mit einer Krankheit eigener Art zu thun haben, welche von den bekannten foetalen Knochen-Erkrankungen durchaus verschieden ist.und sich durch ibr makroskopisches, wie mikroskopisches Verhalten scharf von denselben trennen lässt.

Ueber das Wesen der Krankheit sind wir völlig im Unklaren. Anamnestisch hat sich bis jetat nichts feststellen lassen, und auch aus den mikroskopischen Bildern lässt sich kein Schluss auf die etwaigen Ursachen der Veränderungen ziehen.

1) Kaufmann, Lehrbuch der speciellen patbologischen Anatomie Berlin 1896. 
Man kann die Hypothese aufstellen, dass durch irgend eine Schädlickeit, welche im Blut kreist, die Resorption des Knorpels verzögert und vor Allem die Bildung von Knochensubstanz behindert wird. Die in unserem Falle vorhandenen Nekrosen lassen sich vielleicht so deuten, dass bei verstärkter Einwirkung jener Schädlichkeit die Gewebe so sehr beeinträchtigt werden, dass ein Absterben derselben eintritt.

\title{
$\mathbf{X X}$. \\ Ueber die im Malaischen Archipel vorkommenden Malaria-Erreger nebst einigen Fiebercurven
}

\author{
von \\ Dr. Max Glogner, \\ Stadtgeneesher in Samarang (Java). \\ Hierzu Taf. X.-XI.
}

Während in den letzten Jahren die Erreger der MalariaFieber verschiedener Tropengegenden beschrieben wurden, fehlt bis jetzt eine einigermaassen genaue Mittheilung über den MalariaParasiten aus dem Malaischen Archipel, einem der Hauptheerde der Malaria-Erkrankungen. Ich möchte mit folgenden Zeilen diese Lücke ausfüllen und an der Hand einzelner klinisch beobachteter Fälle über die bereits aus anderen Tropengegenden bekannten, sowie über zwei von mir selbst gefundene Erreger berichten, von denen der eine bereits früher in diesem Archiv Gegenstand einer näheren Beschreibung geweseu ist.

Im Malaischen Archipel kommen nach meinen Beobachtungen 4 verschiedene Malaria-Parasiten vor, welche ich auf den beifolgenden Tafeln wiedergebe. Die Krankheitsfälle, bei welchen diese Parasiten gefunden wurden, zeigten klinisch bisweilen die grösste Aehnlichkeit, während sie verschiedene Erreger behor- 